\title{
FUNCTION AND SAFETY EVALUATION OF 3D TECHNOLOGY TO PREPARE BONE REPAIR BIOMATERIALS
}

\author{
Elcin Huseyn, Research Laboratory of Intelligent Control and Decision-Making Systems in Industry \\ and Economics, Azerbaijan State Oil and Industry University, Baku, Azerbaijan, \\ ORCID ID: https://orcid.org/0000-0001-5965-7419 \\ DOI: https://doi.org/10.31435/rsglobal_conf/28022021/7433
}

\begin{abstract}
PLGA/HA composite biomaterials are prepared, and 3D printing technology is used to make bone scaffolds that can be implanted in the body. Its performance is tested by in vitro physical and biological methods, and its safety is evaluated by animal experiments. Methods: $3 D$ printing technology was used to print the PLGA/HA composite three-dimensional stent biomaterial, and the tensile strength and bending strength of the stent material were tested with reference to GB/T1040 and $G B / T 9341$ to verify its ability to support the proliferation and differentiation of hMSC. The biological evaluation standard (GB/T16886) evaluates the biocompatibility and biosafety of scaffold materials in vitro and in vivo. Results: The porous $3 D$ scaffold made of PLGA/HA composite material was successfully fabricated; the mechanical tensile strength and flexural strength of the composite material were $38 \mathrm{MPa}$ and $42 \mathrm{MPa}$ respectively, which were 5.35 times and 5.25 times that of normal human cartilage; in vitro cell test It is proved that the $3 D$ scaffold can support the proliferation and differentiation of hMSC into chondrocytes. The results of the biosafety test show that the scaffold meets the national medical device biological evaluation standards.
\end{abstract}

Keywords: Biomanufacturing, biomaterials, differentiation, human bone marrow mesenchymal stem cells.

1. Introduction. Bone tissue damage is a major problem facing human health. With the development of medical science, bone cement materials and artificial bone grafting technology have been widely used in clinical practice, but there are still some problems. For example, organic bone cement has poor biocompatibility and large heat generation during the forming process, which may aggravate the patient's condition; inorganic bone cement will have a slow forming and curing rate in the body and poor flexibility; artificial bone transplantation treatment is always There are problems such as source limitation and possible immune response. Tissue engineering is an important potential alternative treatment measure, which is expected to eventually solve this problem [1].

The purpose of tissue engineering scaffold materials is to provide a three-dimensional (threedimension, 3D) scaffold for cells, which is conducive to cell adhesion, proliferation, and differentiation, and provides suitable environmental support for cell growth. The production of 3D scaffolds plays an especially important role in tissue engineering research, and it is the key to the industrialization of tissue engineering.

Traditional 3D scaffolding techniques include solvent casting-particle leaching method [2], gas foaming method [3], emulsification/freeze-drying method, phase separation method, etc. However, when considering the porosity and pore size of the material, the above the medical recycled materials produced by the method have certain limitations. The use of 3D printers to make stent materials is a hot spot in the international regenerative medicine research [4-5]. Zopf et al. [6] reported the use of a 3D printed tracheal splint for the treatment of tracheobronchomegalies. One year after the treatment, the infant patient grew the left main bronchus. Generally, MRI is used to obtain the three-dimensional data of the patient's bone injury site, the obtained three-dimensional data is converted into a threedimensional printing model by software, compiled into a file format recognized by a 3D printer, and rapid prototyping technology is used to make bone tissue models and tissue scaffolds [7], After adapting the stent material made in vitro and transplanting it into the diseased part of the patient, it may greatly alleviate the patient's illness and even save some patients' lives. Tarafder et al. [8] used the tricalcium phosphate (tricalcium phosphate, TCP) scaffold made by 3D technology and transplanted it into a mouse femoral defect model for treatment and found that it can accelerate the formation of osteocytes in new bone tissue in the early stage. 
This experiment describes a method of rapidly manufacturing polylactic acid-glycolic acid [9]/ hydroxyapatite [poly (lactide-co-glycolide)/hydroxyapatite, PLGA/HA] composite 3D scaffold using a 3D printer. The materials were tested for the corresponding mechanical properties, in vitro supported human bone marrow mesenchymal stem cells (human bone marrow derived mesenchymal StemCells, hMSC) proliferation and differentiation test and in vivo biological safety assessment.

\section{Materials and methods}

\subsection{Material}

\subsubsection{Bracket material}

PLGA (75:25), with a relative molecular mass of 100,000 was purchased from Istanbul Biotechnology Industrial Co., Ltd.; HA was purchased from Siemens Biotech Company; dioxane was purchased from Asminaros Industry Co., Ltd.

\subsubsection{Equipment and consumables}

The 3D printing equipment was Automation 3200, and the software used was NVVIEWER, provided by the Istanbul Fatih Technical School of Istanbul University; 3D printing containers and needles were purchased from Japan's Hitachi Company; cell incubators were purchased from American Thermoelectric Company; CKX41 inverted microscope system was purchased from Japan Olympus; BHC-1000IIA2 biological safety cabinet was purchased from Jiangsu Sujing Company; desktop centrifuge was purchased from Eppendorf; Quanta450 scanning electron microscope was manufactured by FEI Company of the United States; CS-600B automatic biochemical analyzer produced by Di Rui; Shenzhen Pu Kangchan PE-6800 semi-automatic blood cell analyzer.

\subsubsection{Cells and reagents}

hMSC cells (ATCCPCS-500-012) were purchased from the American Type Culture Collection (American Type Culture Collection, ATCC); cell basal medium (ATCCPCS-500-010M-APCS-500-10H0M-A-PCS-9500) ) Purchased from Saiye (Guangzhou) Biotechnology Co., Ltd.; complete medium and cartilage formation complete medium are basal medium and cartilage formation basal medium containing final concentration of $10 \%$ fetal bovine serum; fetal bovine serum and pancreatin are purchased from Gibco Company; Recombinant human $\beta 1$ a interferon (recombinant human interferon- $\beta 1 \mathrm{a}$, rhIFN- $\beta 1 \mathrm{a}$ ) was prepared by our laboratory; Recombinant human nerve growth factor (recombinant humananervectin II lab anti-collagen, mouse anti-collagen) Collagen II) antibody was purchased from Abcam; Alcian Blue solution was purchased from Sigma Aldrich; nuclear fast red counterstaining kit was purchased from Shanghai Jiemei Company.

\subsubsection{Experimental animals.}

Healthy big-eared white rabbits, male or female, weighing 1.8 to $2.0 \mathrm{~kg}$, provided by Istanbul Institute of Biological Products Co., Ltd.

\subsection{Method}

\subsubsection{Preparation and pretreatment of scaffold materials.}

PLGA is divided into 3 groups. Weigh $1.2 \mathrm{~g}, 1.8 \mathrm{~g}$ and $3.0 \mathrm{~g}$ of PLGA respectively, add them to $5 \mathrm{ml}$ of dioxane, and dissolve them completely; each group has a fixed HA mass of $3 \mathrm{~g}$, add an appropriate volume of dioxane Infiltrate and stir to make it completely infiltrated; each group will mix and stir the dissolved PLGA and HA evenly.

\subsubsection{D bracket printing and post-processing.}

Print a 3D bracket of $4 \mathrm{~cm} \times 4 \mathrm{~cm} \times 2 \mathrm{~cm}$, the $3 \mathrm{D}$ printing equipment is Automation 3200; set the printing parameters of the 3D material bracket: the material between layers is stacked vertically and crosswise, and each layer is placed in parallel, with a gap between each strip $2 \mathrm{~mm}$, the step height of each step in the $\mathrm{Z}$ axis direction is $0.2 \mathrm{~mm}$, the movement speed items in the $\mathrm{X}$ axis and $\mathrm{Y}$ axis directions are $2 \mathrm{~mm} / \mathrm{s}$, the aperture of the discharge port is controlled at $0.5 \sim 0.1 \mathrm{~mm}$, the discharge port The pressure is controlled at 30 to 60 psi. Put the pre-processed stent material into the raw material storage room of the printing equipment, run the program, and print out the designed threedimensional stent according to the set program. During the printing process, the temperature of the stent platform is maintained at $-20 \sim-10^{\circ} \mathrm{C}$, and cold air is continuously applied to separate the solid and liquid phases. After the stent is printed, it is vacuum freeze-dried.

\subsubsection{Material mechanical performance testing.}

The tensile strength and flexural strength of the three prepared materials were tested by Istanbul Material Surface Analysis and Testing Center. The testing instrument was a universal electronic testing machine, and the testing methods were GB/T1040 and GB/T9341. 


\subsubsection{Cutting and pretreatment of $3 D$ stent.}

The 3D material is laser cut into small pieces of $1 \mathrm{~cm} \times 1 \mathrm{~cm} \times 2 \mathrm{~cm}$. After being sterilized by $\gamma$ ray radiation, the sealed package is placed in a suitable aseptic container. The material is completely immersed in $75 \%$ ethanol, soaked overnight, and the ethanol is discarded. Wash with sterile saline for 3 times, place the material in a suitable sterile container, add $5 \mathrm{ml}$ of saline at $37^{\circ} \mathrm{C}$ for 24 hours, and use the extract and material for pyrogen experiment and subcutaneous implantation experiment in safety evaluation. Before the scaffold material is used in the cell differentiation experiment, the material needs to be soaked in the complete medium for 24 hours. After taking it out, the residual solution is blotted with sterile filter paper and then inoculated with hMSC.

\subsubsection{Cultivation and inoculation of hMSC cells.}

The hMSC cells were cultured in T175 gas-permeable cell flasks and cultured with complete medium. After the cells covered the bottom of the flask, $2 \mathrm{ml}$ of trypsin was added for $1 \mathrm{~min}$, and $10 \mathrm{ml}$ of complete medium was added to terminate the trypsinization reaction, and the cell suspension was collected in a centrifuge tube After centrifugation, the cells were collected, the supernatant medium was discarded after centrifugation, and an appropriate amount of complete medium was added to resuspend the cells to a density of $2 \times 106 \mathrm{cel} / \mathrm{ml}$. Place the aseptically treated 3D scaffold in a 6-well plate, drop the cells dropwise to the center of the material, add $0.5 \mathrm{ml}$ of each material dropwise, let it stand for 1 hour, then add $3 \mathrm{ml}$ of complete medium and place it at $37^{\circ} \mathrm{C}$, Culture in a $5 \% \mathrm{CO} 2$ incubator.

2.2.6. Electron microscopic detection of hMSC proliferation and osteogenic differentiation on the scaffold.

After the hMSC was inoculated on the scaffold material, the material was taken on the 7th day, and the material was washed with phosphate buffered saline (PBS), fixed with 3\% glutaraldehyde, dehydrated by ethanol series, critical point drying, sprayed with gold, and observed by scanning electron microscope. Proliferation of adhesion on the stent. At the same time, the scaffold material without cells was observed under electron microscope.

\subsubsection{Chondrogenic induction and differentiation of hMSC cells to be cells.}

After culturing on the 3D material for 2 to 3 days, remove the complete medium. Complete medium was added to the negative group, and complete cartilage medium was added to the positive control group. In the positive group, the complete cartilage medium was replaced every 3 days, and differentiation was induced for 21 days. The negative group was replaced with complete medium every 3 days for a total of 21 days.

\subsubsection{Glycosaminoglycans derived from osteogenic differentiation of hMSCs} (glucosamine glycan GAG).

Assay: Remove the cartilage-forming complete medium from the positive group of the 6-well plate, wash the material twice with PBS, transfer the material to a new 24-well plate, add $0.2 \mathrm{ml}$ of trypsin to the surface of the material, and digest the cells for 2 minutes. Then add $1 \mathrm{ml}$ of complete medium to wash the cells in the material into a 24 -well plate, and incubate in a $37^{\circ} \mathrm{C}, 5 \% \mathrm{CO} 2$ incubator for 72 hours. On the third day, carefully remove the medium from the wells, add cleaning solution to wash, incubate in acidic solution for 3 minutes, treat with AlcianBlue staining solution for 30 minutes at room temperature, wash twice, and observe under an inverted microscope after nuclear fast red counterstaining. The negative control was also processed according to the above method.

\subsubsection{Western blot detection of chondrocyte specific marker collagen II}

Trypsin digests the cells on the material, add $1 \mathrm{ml}$ of complete medium to stop the trypsin reaction. After counting, each group is centrifuged to collect $1.0 \times 105$ cells, discard the supernatant, add $20 \mu 1$ of cell lysate, and centrifuge for $10 \mathrm{~min}$. Afterwards, take the supernatant, add $2 \times$ protein loading buffer, mix and heat, load the sample for denaturing polyacrylamide gel electrophoresis. After electrophoresis, the protein on the separation gel is transferred to the polyvinylidene fluoride membrane, the blocking solution is blocked, the anti-collagen II antibody is incubated, the enzyme-labeled secondary antibody is incubated, and the substrate color developing solution is added for color development.

\subsubsection{Safety assessment of 3D scaffold materials in animals}

The pyrogen and subcutaneous implantation of 3D stents are tested according to the current biological evaluation standards of medical devices and relevant standards such as the Pharmacopoeia of the People's Republic of China (2010 Edition). There are 3 animals in parallel for each experiment, and the average value of the experiment is taken. 


\subsubsection{Pyrogen experiment}

Before the experiment, select 3 rabbits whose highest and lowest body temperature does not exceed $0.4{ }^{\circ} \mathrm{C}$. For the preparation method of the physiological saline extract of the test material, see 2.2.4. Slowly inject the extract that has been preheated to $38^{\circ} \mathrm{C}$ through the ear vein at a dose of $10 \mathrm{ml} / \mathrm{kg}$ to ensure that the injection is completed within $15 \mathrm{~min}$. After the injection, the body temperature was measured every 1 hour for a total of 3 times. The highest value of the rectal temperature minus the basal body temperature is the body temperature rise value.

\subsubsection{Subcutaneous implantation test}

For the treatment of the tested materials, see Method 2.2.4. Take 3 healthy New Zealand white rabbits, remove the coat on the test site on the back of the rabbits. After disinfection, prepare a subcutaneous sac at the skin incision site $2 \mathrm{~cm}$ away from both sides of the spine by blunt dissection. The bottom of the sac should be at least $1 \mathrm{~cm}$ away from the skin incision. The test material block was implanted under the skin, the skin was sutured, and kept for 12 weeks. During this period, observe and record the rabbit's general condition, activities, diet, drinking, weight gain, etc., before and 4 weeks after subcutaneous implantation., 8 weeks, 12 weeks, weighed, blood samples were collected from the ear veins, blood routine indexes and serum biochemical indexes were measured.

\section{Experimental results}

\subsection{D material printing}

The printer prints the 3D stent material according to the above procedure and can print out the 3D stent whose appearance and structure meet the expectations. After the later vacuum freeze-drying, the 3D stent material as shown in Figure 1 can be obtained.

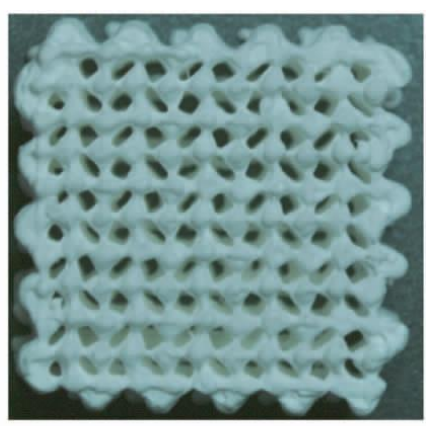

(a)

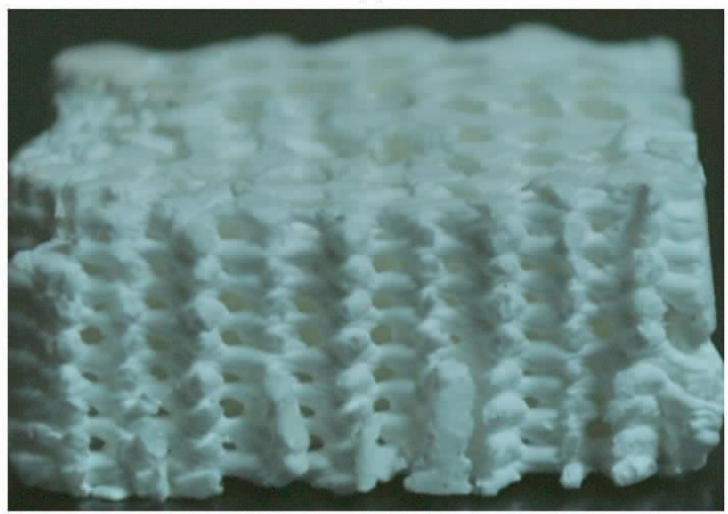

(b)

Fig. 1. Photo of 3D scaffold

Bird's eye view (a) and View of longitudinal section (b)

\subsection{Testing of mechanical properties of PLGA/HA composite materials.}

The measured values of the tensile strength and elastic strength of the PLGA/HA composite material mixed in different proportions are shown in Table 1. Among them, the material with PLGA: HA ratio of 3:5, its tensile strength and elastic strength are 5.35 times and 5.25 times of the average value of human cartilage. This group is selected for further testing. 
Table1. The strength detection result of PLGA/PLA composite material.

\begin{tabular}{|c|c|c|c|c|}
\hline PLGA: HA & $2: 5$ & $3: 5$ & $5: 5$ & Human cartilage [10] \\
\hline Tensile performance (MPa) & 24 & 38 & 45 & $3.7 \sim 10.5$ \\
\hline Bending strength (MPa) & 35 & 42 & 58 & $0.7 \sim 15.3$ \\
\hline
\end{tabular}

\subsection{Electron microscope results of cell attachment and proliferation of scaffold materials.}

After the material is processed, observe the growth of the cells under an electron microscope, as shown in Figure 2. Figure 2 (a) is an electron microscope image of the material without cells. The surface of the material has small holes and roughness; Figure 2 (b) is the hMSC inoculated on the material, the cells are attached, the surface of the material is smooth, the connections between the cells are smooth, and the cells in the stretched state can be seen, and the cell growth is in good condition.

\subsection{Proteoglycan detection.}

Results After proteoglycan staining, the cells in the negative group showed a long spindle shape, and no blue glycoprotein appeared around the cells, as shown in Figure 3 (a).

The positive group has obvious changes in cell morphology, and many proteoglycans appear around the cells, as shown in Figure 3 (b).

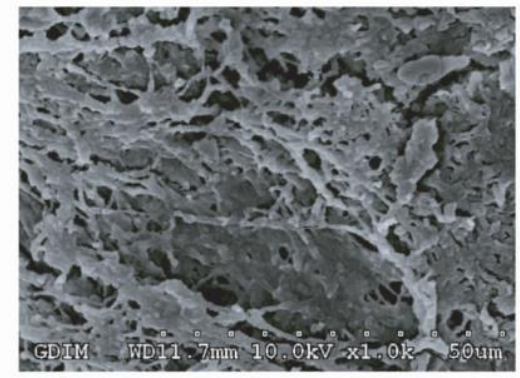

(a)

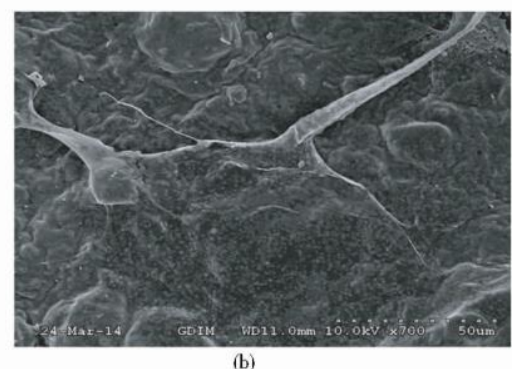

Fig. 2. SEM photo of blank biomaterial (a) and SEM photo of hMSC on biomaterial ( $b$ )

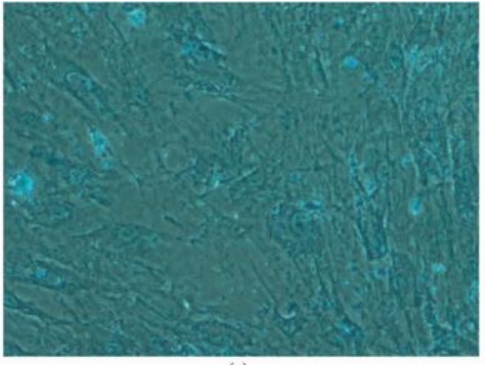

(a)

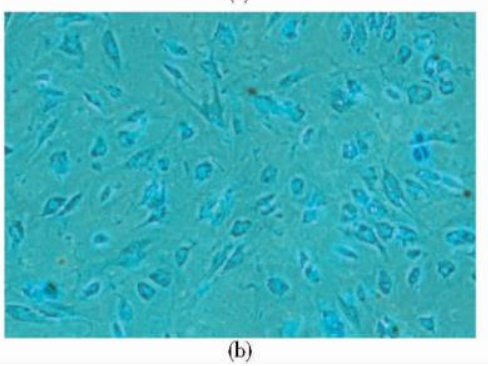

Fig. 3. GAG decision photo of MSC on biomaterial

Negative control (a) and Positive control (b) 
3.5. Western blot detection results of Collagen II. The cells of the negative group and the positive group were detected by immunoblotting of type II collagen. The results showed that the immune bands of collagen II appeared in each group, the negative group was the lowest, and the expression level of collagen II in the positive group was higher than that in the negative group. The gray scale analysis value proved that the relative expression of the positive group was significantly different than that of the negative group $(\mathrm{P}<0.01)$. It shows that the scaffold material can promote the differentiation of hMSC into chondrocytes, as shown in Figure 4.

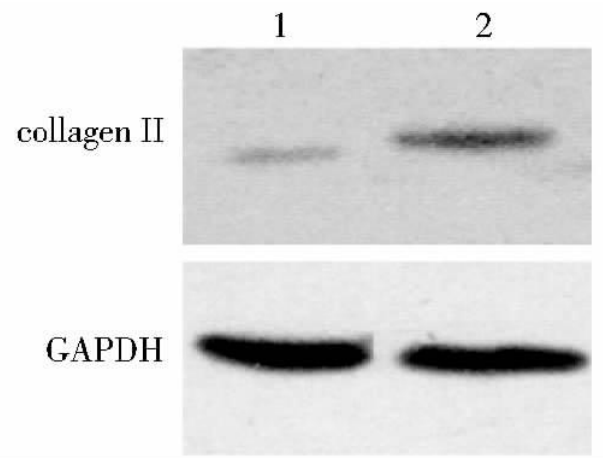

Fig. 4. Western blot result of collagen II of different experimental groups Negative control (1) and Positive control (2)

3.6. Results of pyrogen experiment. In the 3 rabbits tested, the body temperature rise was lower than $0.6^{\circ} \mathrm{C}$, and the total body temperature rise of the 3 rabbits was lower than $1.4^{\circ} \mathrm{C}$. The pyrogen inspection of the test product PLGA/HA stent complied with national regulations. The test results are shown in Figure 5.

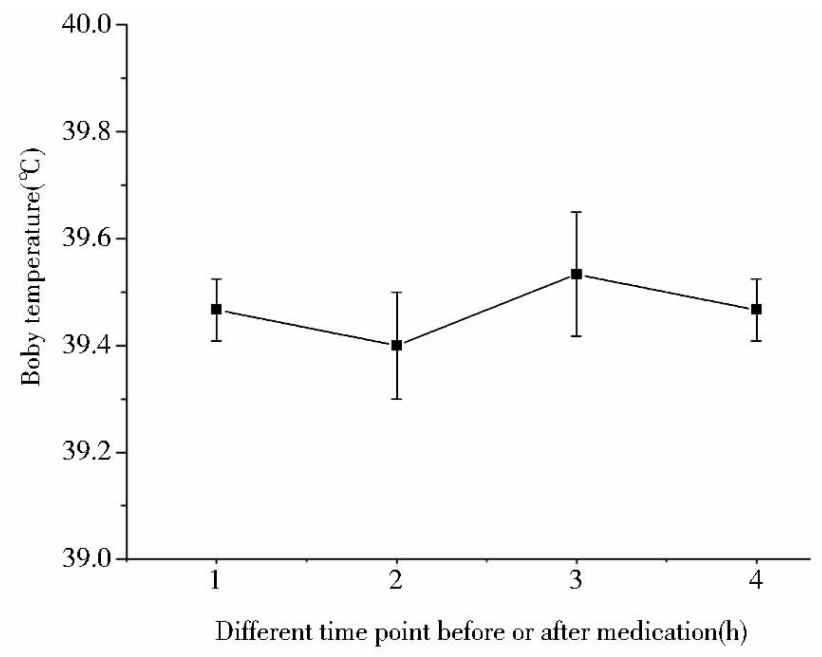

Fig. 5. Pyrogen test experiment samples based on animal method.

$0.5 \mathrm{~h}$ before medication (1), $1 \mathrm{~h}$ after medication (2), $2 \mathrm{~h}$ after medication (3) and $3 \mathrm{~h}$ after medication (4)

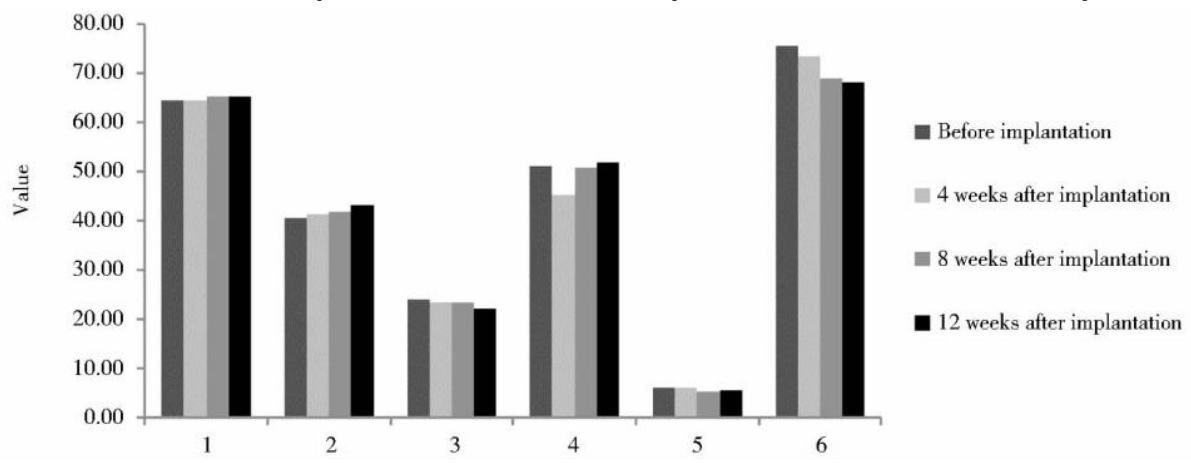

Fig. 6. Serum biochemical values of experimental animal

Serum total production $(g / L)(1)$, Albumin $(g / L)(2)$, Globulin $(g / L)(3)$,

Alanine transaminase (U/L) (4), Urea nitrogen (mmol/L) (5) and Creatinine ( $\mu \mathrm{mol} / \mathrm{L})(6)$. 


\subsection{Test results of subcutaneous implantation test}

For the treatment of the scaffold material of the test product, refer to Method 2.2.4. During 12 weeks after the test material was implanted subcutaneously in the rabbit, there was no abnormality in its general condition, activity, diet, drinking, weight gain, etc.; its serum biochemical indicators and transplantation There was no obvious abnormality in the comparison before entry, the test result is shown in Figure 6; the blood routine index has no obvious abnormality compared with that before the implantation, and the test value is shown in Figure 7. After 12 weeks of implantation, the implanted test material block has been degraded and absorbed There is no purulent or secretion in the surrounding tissues of the implantation site. The pathological examination results of the surrounding tissues of the material show that they are all normal tissues (results not shown). It shows that the animal subcutaneous implantation test of the scaffold material of the test product meets the requirements.

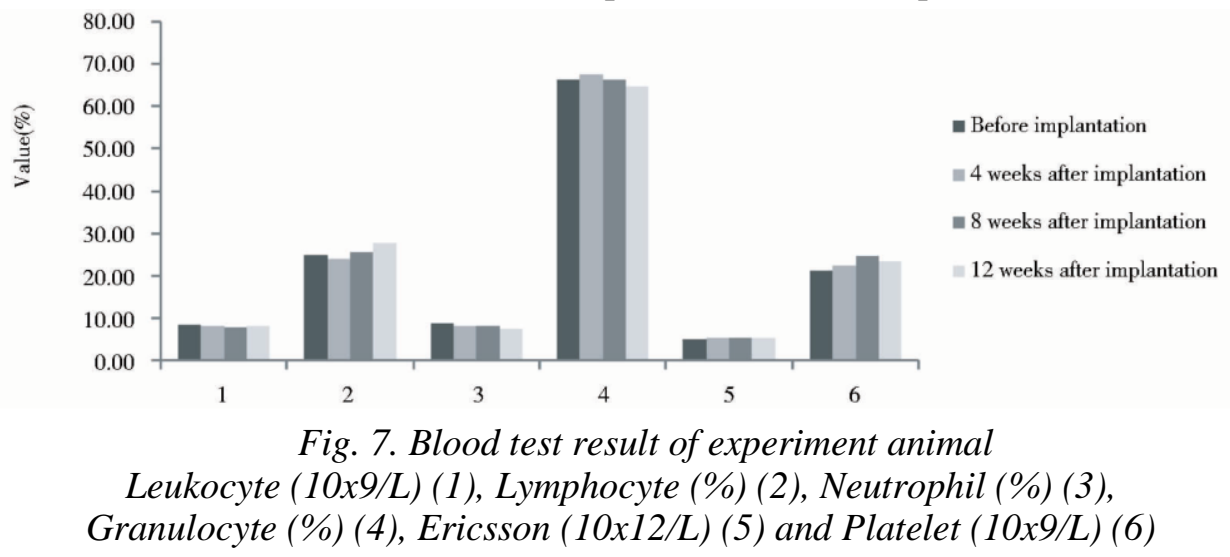

4. Discussions. PLGA is a biodegradable material approved by FDA for use in the body. It can be gradually degraded over time without affecting the growth of fresh bone tissue. The grid-like topology facilitates the formation of blood vessels and the exchange of body fluids. The degradation products are brought into the body fluid circulation to facilitate the growth and differentiation of cells and the formation of new bone tissue. Attawia et al. [11] used a three-dimensional PLGA/HA (50:50) composite scaffold prepared by a particle leaching method. After being planted with bone cells, the bone cells can adsorb and proliferate perfectly. In this experiment, PLGA $(75: 25)$ was selected, with a relative molecular mass of 100,000, which ensures that the material made has a degradation cycle of 2 to 3 months in the body [10] and does not affect the production of fresh bone tissue. According to the different application functions of the stent material, the appropriate proportion of PLGA is selected to achieve the corresponding application effect [12].

The 3D printing technology mainly compiles three-dimensional material parameters through software and realizes the printing of materials through precise control of the printer, while the solidification and molding of materials rely on different molding mechanisms. In the literature of bone repair materials, polymer liquid solidification technology or fused deposition technology is often used in combination with a $3 \mathrm{D}$ printer to form $3 \mathrm{D}$ printing of bone repair materials. This experiment uses a traditional phase separation molding technology combined with a 3D printer. The specific process is to dissolve and mix inorganic materials and organic materials in a volatile organic solvent to form a liquid with a certain viscosity, spray it through a pressure-controlled nozzle to a work surface with a surface temperature of about $-15^{\circ} \mathrm{C}$, and give the work surface a certain amount. With strong cold air, the organic materials and electrodeless materials in the sprayed liquid will quickly solidify, the solvent will evaporate, the solid phase and the liquid phase will separate, and finally a preset 3D scaffold will be formed [13]. After the molded 3D material is freeze-dried to remove residual organic solvents, it will eventually become the required scaffold material that can be used for experiments.

The mechanical strength of the scaffold material was tested. The measured values of tensile strength and bending strength of the mixed material were $38 \mathrm{MPa}$ and $42 \mathrm{MPa}$, which were 5.35 times and 5.25 times the strength of human cartilage [10], both of which were greater than the strength of human cartilage. Higher strength may meet the needs of clinical trials. On the one hand, the strength of the skeleton will decrease with the degradation of the components, and the addition of inorganic HA may promote the reduction of the strength of the stent. Therefore, in order to ensure that the PLGA/HA scaffold material maintains a specific three-dimensional structure during cell culture and in vivo experiments, and to ensure that the decrease in its autogenous strength can be compensated by the strength of new bone 
deposition, its mechanical properties before degradation are higher than the corresponding human bone mechanics Performance is a necessary condition for the degradation of bone materials. Giordano et al. [14] used a 3D printer to prepare a PLA (relative molecular weight of 230,000) three-dimensional scaffold material, and its bending strength was $93 \mathrm{MPa}$ and $206 \mathrm{MPa}$ respectively after 48 hours of preparation and one week after freeze-drying, but its tensile strength was only respectively $10 \mathrm{MPa}$ and $11 \mathrm{MPa}$. The flexural strength of the organic material scaffold prepared by Giordano et al. is much greater than the tensile strength, which is determined by the characteristics of the material itself. The mixed materials selected in this experiment make the prepared materials have the flexibility of organic materials and the flexibility of inorganic materials. Hardness, the clinical application effect will be more effective.

In this experiment, the inducing substance used in the chondrogenic differentiation control group was the classic TGF $\beta 3$ [15]. In addition, other cytokines or proteins have been tried to induce chondrogenesis [16], such as rhNGF, thymosin, and rhIFN $\beta$. Through experiments, it is found that TGF- $\beta 3$ combined with certain cytokines can better promote the chondrogenic differentiation effect of hMSC. Subsequent experiments will further study the mechanism of the combined use of cytokines to induce differentiation.

According to the current biological evaluation standards for medical devices promulgated by the State Food and Drug Administration and relevant standards such as the pharmacopoeia, this experiment has tested the contents of pyrogen, hemolysis, systemic acute toxicity, intradermal irritation, subcutaneous implantation, etc. (only part of the experiment). Display) for testing (GB/T16886, Biological Evaluation of Medical Devices). The results of animal experiments showed that the pyrogen test, hemolysis test, systemic acute toxicity test, intradermal irritation test, subcutaneous implantation test and other contents of the tested stent material all meet the biological evaluation standards of medical devices. It shows that the method of making 3D scaffold materials in this experiment is feasible. Later experiments will conduct pharmacodynamic tests of scaffold materials in animals to further verify the function of 3D scaffold materials prepared by this method.

\section{REFERENCES}

1. Wang D.L. The birth and development of tissue engineering. Chinese Journal of Biological Engineering, 2014, 34(5): 122-129.

2. $\quad$ Chen S.S., Yang Q., Shen X.Y., et al. Solvent casting-particle leaching method to prepare PBS/PCL tissue engineering scaffold. Journal of Donghua University, (Natural Science), 2009, 35(4): 391-395.

3. Huang Y.C, Mooney D.J. Gas Foaming to Fabricate Polymer Scaffolds in Tissue Engineering. In: Ma P.X., Elise J. Scaffolding in Tissue Engineering. Boca Raton: CRC Press, 2005: 155-167.

4. Murphy S.V., Atala A. 3D bioprinting of tissues and organs. Nature Biotechnology, 2014, 32(8): 773-785.

5. Melchels F., Wingenhauser P., Warne D., et al. CAD/CAM assisted breast reconstruction. Biofabrication, 2011, 3(3): 1-8.

6. Zopf D.A., Hollister S.J., Nelson M.E. Bioresorbable airway splint created with a three-dimensional printer. The New England Journal of Medicine, 2013, 368(21): 2043-2045.

7. Banks J. Adding value in additive manufacturing: Researchers in the United Kingdom and Europe look to 3D printing for customization. IEEE Pulse, 2013, 4(6): 22-26.

8. Tarafder S., Davies N.M., Bandyopadhyay A., et al. 3D printed tricalcium phosphate bone tissue engineering scaffolds: effect of $\mathrm{SrO}$ and $\mathrm{MgO}$ doping on in vivo osteogenesis in a rat distal femoral defect model. Biomaterials Science, 2013, 1(12): 1250-1259.

9. Gentile P., Chiono V., Carmagnola I. An overview of poly (lactic-co-glycolic) acid (PLGA)-based biomaterials for bone tissue engineering. International Journal of Molecular Sciences, 2014, 15(3): 3640-3659.

10. Parsons J.R. Cartilage. In: Black J., Hastings G. Handbook off Biomaterials Properties. New York: Chapman \& Hall, 1998: 40-46.

11. Attawia M.A., Herbert K.M., Laurencin C.T. Osteoblast-like cell adherence and migration through 3-dimensional porous polymer matrices. Biochemical and Biophysical Research Communications, 1996, 213(2): 639-644.

12. Li S.M. Hydrolytic degradation characteristics of aliphatic polyesters derived from lactic and glycolic acids. Journal of Biomedical Materials Research, 1999, 48(3): 342-353.

13. Liu L., Xiong Z., Yan Y.N., et al. Porous morphology, porosity, mechanical properties of poly ( $\alpha$-hydroxy acid)-tricalcium phosphate composite scaffolds fabricated by low-temperate deposition. Journal of Biomedical Materials Research, 2007, 82A (3): 618-629.

14. Giordano R.A., Wu B. M., Borland S.W., et al. Mechanical properties of dense polylactic acid structure fabricated by three-dimensional printing. Journal of Biomaterials Science, Polymer Edition, 1996, 8(1): 63-75.

15. Indrawattana N., Chen G.P., Tadokoro M., et al. Growth factor combination for chondrogenic induction from human mesenchymal stem cell. Biochemical and Biophysical Research Communications, 2004, 320(3): 914-919.

16. Cicione C., Muinos-Lopez E., Hermida-Gomez T. Alternative protocols to induce chondrogenic differentiation: transforming growth factor- $\beta$ superfamily. Cell and Tissue Banking, 2014, 12(2): 195-207. 